\title{
Different Factors Correlated to Early Rebleeding in Cirrhotic Patients Treated by Variceal Band ligation versus Endoscopic Sclerotherapy
}

\author{
Kamal Amer, Amal Jouda, Sameh M. Abdel Monem
}

Tropical Medicine Department, Faculty of Medicine, Zagazig University, Egypt

Corresponding Author Kamal Amer

Mobile:

+201226204876

\section{E mail: \\ dr_kamalamer@yahoo .com}

Key words: oesophageal varices, endoscopic sclerotherapy, endoscopic band ligation
Background and study aim : Endoscopic treatment has become the principal first-line intervention in patients with bleeding oesophegeal varices, both during the acute event and for long-term therapy to prevent recurrent bleeding. Several clinical considerations affect the prognosis in individual patients including the severity portal hypertension, the location of the bleeding varices, residual hepatic function, the presence of associated systemic disease, and others. Early rebleeding has been shown to be a strong predictor of mortality and recurrent variceal bleeding substantially increases the risk of complications which further contribute to mortality. This study aimed to evaluate early rebleeding after different methods of endoscopic intervention and investigate the different parameters of the patient that can be correlated to it.

Patients and methods: Hundred and four cirrhotic patients with first attack of variceal bleeding were included in this study. They were randomly allocated to two groups, group I: 52 patients who were managed by endoscopic variceal sclerotherapy and group II: 52 patients who were managed by endoscopic variceal band ligation to control their attack. The patients were followed up for six weeks and all their clinical, laboratory, endoscopic parameters were monitored. The rate of mortality and

\section{INTRODUCTION}

Portal hypertension commonly complicates liver cirrhosis and the development of oesophegeal varices is one of the major complications of portal hypertension [1]. The prevalence of oesophegeal varices at diagnosis ranges from $0-10 \%$ in patients with compensated cirrhosis, to $60 \%$ to $80 \%$ in patients with decompensated cirrhosis and the reported mortality from variceal early rebleeding was measured and correlated to these different patients' parameters

Results: There was no significant difference between the two groups as regards rate of early rebleeding $(15.4 \%$ in group I vs $9.6 \%$ in group $\mathrm{II} \mathrm{P}=0.374)$. The rate of early rebleeding was significantly correlated to Child's score ( $\mathrm{r}=+0.136 \mathrm{P}=0.014)$, PT $(\mathrm{r}=+0.35 \mathrm{P}<0.001), \mathrm{INR}(\mathrm{r}=+0.419 \mathrm{P}<0.001)$, grade of $\mathrm{OV}(\mathrm{r}=+0.233 \mathrm{P}=0.001)$, risky signs $(\mathrm{r}=+0.179 \mathrm{P}=0.001)$, units of blood received $(\mathrm{r}=+0.387 \mathrm{P}<0.001)$, amount of ethanolamine oleate $(\mathrm{r}=+0.329 \quad \mathrm{P}=0.017)$ and number of rubber bands used ( $\mathrm{r}=$ $+0.245 \mathrm{P}=0.039$ ). Mortality rates showed also no significant difference during the six weeks of follow up , (19.2\% in group I vs $21 \%$ in group II $\mathrm{P}=0.647)$, as well as mortality rates in rebleeding cases $(37.5 \%$ in group I vs $40 \%$ in group II P=0.925).

Conclusion: The factors that are strongly correlated to rate of early rebleeding after endoscopic management of $\mathrm{OV}$ are severely decompensated liver disease, larger OV size and presence of risky signs, use of more blood units during resuscitation, use of large amount of ethanolamine oleate during sclerotherapy and use of more rubber band during banding. Sclerotherapy and band ligation are comparable to each other in most outcomes especially early rebleeding and mortality. 
After control of the index bleed, there is a $40 \%$ chance of rebleeding with a similar mortality. The risk of rebleeding is greatest during the first few days after initial variceal hemorrhage [5]. Survival after variceal bleeding depends largely on the rapidity and efficacy of initial primary hemostasis and the presence and severity of underlying liver disease and hepatic functional reserve [6].

Early rebleeding has been shown to be a strong predictor of mortality and recurrent variceal bleeding substantially increases the risk of complications which further contribute to mortality [6]. Rapid and sustained control of variceal bleeding remains the principal imperative of endoscopic intervention [7]. Several important clinical considerations influence the prognosis in individual patients. These include the natural history of the disease causing the portal hypertension, the location of the bleeding varices, residual hepatic function, the presence of associated systemic disease, continuing drug or alcohol abuse, patency of major splanchnic veins and the response to each specific treatment [8].

Until now, there has been no general consensus on the risk factors and measures to prevent early rebleeding in cirrhotic patients in Egypt. Variceal Band ligation and Endoscopic Sclerotherapy can be effective methods to manage variceal bleeding and may be prevent it primarily and secondarily. However, early recurrent bleeding as a vital complication after variceal band ligation and endoscopic sclerotherapy has not been studied fully.

\section{Aim of the work:}

The aim of the present study was to evaluate the different factors that can affect the rate of early rebleeding of early rebleeding after different endoscopic treatments of variceal bleeding which help better management of variceal bleeding.

\section{PATIENTS AND METHODS}

This prospective randomized study was conducted in the Intensive Care Unit (ICU), Inpatient and Endoscopy Units of Tropical Medicine Department, Faculty of Medicine Zagazig University, during the period from October 2012 to October 2014. It included 104 patients with first attack of hematemesis and melena diagnosed as bleeding esophageal varices by upper endoscopy. The Sample size was calculated using Epi info version 6.04.

They were divided into 2 groups (age, sex and severity of liver disease matched):

-Group I: included 52 patients who were treated by endoscopic sclerotherapy.

-Group II: included 52 patients were treated by endoscopic band ligation.

\section{Inclusion criteria:}

1- Presence of liver cirrhosis, the diagnosis of cirrhosis was based on clinical, biochemical and ultrasonographic findings with ChildPugh grading.

2- First attack of upper GIT bleeding, which was proven by upper GIT endoscopy as bleeding esophageal varices.

\section{Exclusion criteria:}

1- Patients $<18$ and $>60$ years old

2- Patients who refuse participation in this study.

3- Hepatic patients with other causes of upper GIT bleeding than esophageal varices.

4- Patients with bleeding gastric varices.

5- Patients with recurrent attacks of bleeding oesophegeal varices.

6- Patients with intra or extrahepatic malignancy.

7- Patients who had uncontrolled bleeding for $24 \mathrm{~h}$ after endoscopic treatment.

\section{All patients were subjected to the following:}

1. Thorough medical history taking including:

2. Thorough clinical examination including:

3. The following laboratory investigations:

- Complete blood picture (haemoglobin level, red blood cell count, white blood cell count and platlet count)

- Biochemical liver tests on including: Total and direct serum bilirubin in mgldl, Total serum protein and serum albumin in gmldl, Serum Aspartate amino Transferase (AST) and serum Alanine amino Transferase (ALT) (IULL), Prothrombin time in seconds and international randomization ratio (INR).

- Kidney function tests including blood urea and serum creatinine.

- Serum Bilharzial antigen: using ELISA/ soluble egg antigen (SEA)

- AST platelet ratio index (APRI): APRI = (AST/upper limit normal) x 100/platelet count. Score $<0.5$ excludes fibrosis.Score $>2$ suggests fibrosis.[9] 
- Abdominal Ultrasonography : All the patients were examined using esaotemylab device. They were examined according to the standard maneuvers. Color Doppler ultrasound: All measurements were done by a single radiologist using color Doppler sonography with subjects in the supine or left lateral position. A Power Vision SSA380A system (Esaotemy lab device) with (3 to $5 \mathrm{MHz}$ ) convex and sector pulsed probes. Sonographic examinations were carried out 8 hours after the last meal. In our study, we measured two parameters by Doppler ultrasound:

- Portal vein velocity $(\mathrm{cm} / \mathrm{sec})$ PVV was measured directly using color Doppler ultrasound.

- Hepatic artery resistive index $(\mathrm{RI})=($ peak systolic velocity - end diastolic velocity) / peak systolic velocity [10].

4. Child-Pugh classification for all patients into: $\mathrm{A}, \mathrm{B}$, and $\mathrm{C}$ class according the severity of cirrhosis [11] :

\begin{tabular}{|l|c|c|c|}
\hline \multicolumn{1}{|c|}{ Measure } & 1 point & 2 points & 3 points \\
\hline Total bilirubin, (mg/dl) & $<2$ & $2-3$ & $>3$ \\
\hline Serum albumin, g/dl & $>3.5$ & $2.8-3.5$ & $<2.8$ \\
\hline Pt (seconds prolonged) & $0-4$ & $4-6$ & $>6$ \\
\hline Ascites & None & Mild & Moderate to Severe \\
\hline Hepatic encephalopathy & None & $\begin{array}{c}\text { Grade I-II (or suppressed } \\
\text { with medication) }\end{array}$ & $\begin{array}{c}\text { Grade III-IV (or } \\
\text { refractory) }\end{array}$ \\
\hline
\end{tabular}

\begin{tabular}{|c|c|}
\hline Points & Class \\
\hline $5-6$ & A \\
\hline $7-9$ & B \\
\hline $10-15$ & C \\
\hline
\end{tabular}

\section{Upper gastrointestinal endoscopy : Before endoscopy:}

- Patients admitted to the Intensive Care Unit (ICU) for the first attack of variceal bleeding. Initial resuscitation following the classic Airway, Breathing, Circulation scheme was done followed by nasogastric lavage to remove particulate matter, fresh blood, and clots from the stomach to facilitate endoscopy.

- The patients were given the following medications along with volume replacement with plasma expanders: vitamin $\mathrm{K} 10 \mathrm{mg} /$ day IM, pantoprazole $40 \mathrm{mg} / 12 \mathrm{~h} \mathrm{IV}$, Somatostatin analogue (sandostatin ) initial bolus $500 \mu \mathrm{g}$ iv followed by $250 \mu \mathrm{g} /$ hour for 24 hours and prophylactic antibiotic (cefotaxime sodium 1 gm IV /12h)

At time of endoscopy: Endoscopy was done by a single experienced endoscopist using end flexible videoendoscope (PENTAX VIDEO unit of endoscopy). The patients were positioned on their left lateral position, with head supported on a small firm pillow to remain in a comfortable neutral position and a bite guard in their mouth. Medazolam I.V. was used as sedation. Patients meeting the inclusion criteria were randomized alternatively to undergo Endoscopic Injection Sclerotherapy (EIS) or Endoscopic variceal Band Ligation (EBL).

- EIS was performed using a 25-gauge disposable injection needle for intravariceal and paravariceal injection. The sclerosant used was ethanolamineolyte.

- EVL was performed with PENTAX EG endoscope by the same experienced endoscopist using endoscopic ligating devices: an over tube or multi-band ligators.

\section{Esophageal varices were graded into 4 grades as follows: [12]}

- Grade I: small straight cords of varices continued to lower $1 / 3$ of the esophagus.

- Grade II: moderate sized clubbed varices with well-defined areas of normal mucosa between them, forming several distinct vertical cords and confined to lower third of esophagus.

- Grade III: gross varices extending into the proximal half of the esophagus, which is so large and tortuous, that normal mucosa may 
not be visible in between unless the esophagus is fully distended with air.

- Grade IV: varices are like those of grade III but with dilated capillaries on top or in between varices, (varix over varix).

Portal hypertensive gastropathy was classified as follows: [13]

- PHG grade I: mild reddening and congestive mucosa, no mosaic like pattern.

- PHG grade II: Severe redness and a fine reticular pattern separating the areas of raised edematous mucosa (mosaic like pattern) or fine speckling.

- PHG grade III: Point bleeding + grade II.

\section{After endoscopy:}

The patients used non selective beta blocker carvidalol for prevention of recurrent variceal bleeding, starting with $12.5 \mathrm{mg}$ orally single daily dose as recommended by Banares et al. [14].

The patients were evaluated according to the presence or absence of the following symptoms: epigastric pain, heart burn, retrosternal chest pain, dysphagia, dyspepsia, and odynophagia upon discharge and during the follow up visits every two weeks.

\section{Follow up:}

The follow up of the patients was done every 2 weeks for 6 weeks as regards the following:

1- The patients' general condition like development or improvement of ascites, lower limb edema, jaundice, and hepatic encephalopathy (HE).

2- Development of infections e.g diarrhea, chest infection, and abdominal pain and tenderness as indicators for SBP.

3- Laboratory tests; $\mathrm{CBC}$, total and direct bilirubin, serum albumen and serum creatinine.

4- Upper GIT endoscopy with commenting on the variceal condition as previous, PHG, bleeding and development of sclerosant or post banding ulcer.

5- Rebleeding.

\section{Statistical analysis:}

Data were checked, entered and analyzed using SPSS version 19 EPI-INFO 6 and for data processing and statistic. Numerical data were expressed as mean and standard deviation and the comparison between numerical data is done with simple $\mathrm{t}$ test for normally distributed data and with Mann Whitney $U$ test when data distribution is skewed. We used number and percentage to express categorial data and chisquare test to compare them. The correlation between numerical data was done by Spearman's correlation coefficient. The correlation between numerical and categorical data used Spearman's rank correlation.

\section{RESULTS}

Comparison between the two studied groups as regards age, gender distribution, incidence of diabetes, hypertension and bilharziasis revealed no significant differences as shown in table (1). Table (1) shows also that there were no significant differences as regards the cause of cirrhosis and the previous use of primary prophylaxis.

Table (2) shows that there were no significant differences between the studied groups as regards the liver and spleen size as detected by sonography. There were also no significant differences between the two studied groups as regards portal vein diameter and velocity as well as hepatic artery resistive index measured by coloured doppler, as shown in table (2). Table (2) also shows that there were no significant differences between the two groups as regards all laboratory parameters.

Comparison between the studied groups as regards the endoscopic examination revealed no significant differences between the two groups as regards grade of OV, number of cords, grade of PHG and incidence of duodenopathy at the beginning of the study as shown in table (3).

Table (4) compares the studied groups as regards the incidences of the common post-endoscopy symptoms encountered by the patients and shows that there were no significant differences as regards any of these symptoms.

Table (5) compares the studied groups as regards rate and causes of rebleeding and mortality rate and shows that there were no significant differences between them. Correlation between the rate of rebleeding and study parameters revealed that the rate of rebleeding has significant positive correlation with Child's score, PT, INR, grade of OV, presence of risky signs, number of units of blood transfused during resuscitation, amount of sclerosing agent and number of rubber bands used as shown in table (6). 
Table (1): Demographic data, cause of cirrhosis and comorbidity

\begin{tabular}{|c|c|c|c|c|c|c|}
\hline & $\begin{array}{c}\text { Group I } \\
\text { No }=52\end{array}$ & $\begin{array}{c}\text { Group II } \\
\text { No=52 }\end{array}$ & Test value & $\mathbf{P}$ & Sig. \\
\hline \multicolumn{2}{|l|}{ Age } & $50.1 \pm 12.1$ & $49.7 \pm 10.6$ & $\mathbf{t}=0.15$ & 0.877 & $\mathrm{NS}$ \\
\hline \multirow[t]{2}{*}{ Gender } & Male & $36(69.2 \%)$ & $33(63.5 \%)$ & \multirow{2}{*}{$\mathrm{X}^{2}=\mathbf{0 . 3 8 8}$} & \multirow{2}{*}{0.534} & \multirow{2}{*}{ NS } \\
\hline & Female & $16(30.8 \%)$ & $19(36.5 \%)$ & & & \\
\hline \multicolumn{2}{|l|}{ Diabetes } & $7(13.5 \%)$ & $8(15.4 \%)$ & 0.078 & 0.78 & $\mathrm{NS}$ \\
\hline \multicolumn{2}{|c|}{ Hypertension } & $4(7.7 \%)$ & $6(11.5 \%)$ & 0.443 & 0.506 & NS \\
\hline \multirow{3}{*}{$\begin{array}{l}\text { Cause of } \\
\text { cirrhosis }\end{array}$} & $\mathrm{HBV}$ & $5(9.6 \%)$ & $6(11.5 \%)$ & 0.102 & 0.750 & $\mathrm{NS}$ \\
\hline & $\mathrm{HCV}$ & $45(86.5 \%)$ & $45(86.5 \%)$ & 0.000 & 1.000 & $\mathrm{NS}$ \\
\hline & others & $2(3.8 \%)$ & $1(1.9 \%)$ & 0.343 & 0.558 & $\mathrm{NS}$ \\
\hline \multicolumn{2}{|c|}{ Positive bilharzial Ag } & $7(13.5 \%)$ & $10(19.2 \%)$ & 0.633 & 0.426 & $\mathrm{NS}$ \\
\hline \multicolumn{2}{|c|}{ Primary prophylaxis } & $9(17.9 \%)$ & $10(19.2 \%)$ & 0.064 & 0.800 & $\mathrm{NS}$ \\
\hline
\end{tabular}

Table (2): Baseline sonographic, Doppler data and Child's score and laboratory data

\begin{tabular}{|c|c|c|c|c|c|c|}
\hline & $\begin{array}{c}\text { Group I } \\
\text { No=52 }\end{array}$ & $\begin{array}{c}\text { Group II } \\
\text { No=52 }\end{array}$ & Test value & $\mathbf{P}$ & Sig. \\
\hline \multirow{3}{*}{ Liver size } & enlarged & $2(3.8 \%)$ & $2(3.8 \%)$ & \multirow{3}{*}{$0.877 \#$} & \multirow{3}{*}{0.645} & \multirow{3}{*}{ NS } \\
\hline & Average & $14(26.9 \%)$ & $10(19.2 \%)$ & & & \\
\hline & shrunken & $36(69.2 \%)$ & $40(67.9 \%)$ & & & \\
\hline \multirow[t]{2}{*}{ Spleen size } & average & $3(5.8 \%)$ & $5(9.6 \%)$ & \multirow{2}{*}{$0.542 \#$} & \multirow{2}{*}{0.462} & \multirow{2}{*}{ NS } \\
\hline & enlarged & $49(94.2 \%)$ & $47(90.4 \%)$ & & & \\
\hline \multicolumn{2}{|c|}{$\begin{array}{l}\text { Portal vein diameter }(\mathrm{cm}) \\
\text { Mean } \pm \text { SD }\end{array}$} & $1.58 \pm 0.21$ & $1.53 \pm 0.21$ & $1.188^{\bullet}$ & 0.238 & $\mathrm{NS}$ \\
\hline \multicolumn{2}{|c|}{$\begin{array}{l}\text { Portal vv velocity }(\mathrm{cm} / \mathrm{sec}) \\
\text { Mean } \pm \text { SD }\end{array}$} & $13.27 \pm 3.84$ & $13.24 \pm 4.11$ & $0.299^{*}$ & 0.765 & $\mathrm{NS}$ \\
\hline \multicolumn{2}{|c|}{$\begin{array}{l}\text { Hepatic aa resistive index } \\
\text { Mean } \pm \text { SD }\end{array}$} & $0.78 \pm 0.07$ & $0.77 \pm 0.06$ & $0.104^{*}$ & 0.917 & NS \\
\hline \multirow{3}{*}{$\begin{array}{l}\text { Child's } \\
\text { grade }\end{array}$} & $\mathrm{A}$ & $0.77 \pm 0.06$ & $10(19.2 \%)$ & \multirow{3}{*}{$X^{2}=2.049$} & \multirow{3}{*}{0.359} & \multirow{3}{*}{ NS } \\
\hline & $\mathrm{B}$ & $9(17.3 \%)$ & $15(28.8 \%)$ & & & \\
\hline & $\mathrm{C}$ & $3057.7 \%)$ & $27(51.9 \%)$ & & & \\
\hline \multicolumn{2}{|c|}{ Hemoglobin (g/dl) } & $8.87 \pm 1.53$ & $9.05 \pm 1.52$ & $0.576 \bullet$ & 0.566 & $\mathrm{NS}$ \\
\hline \multicolumn{2}{|c|}{ WBC's (cellx103/ml) } & $6.20 \pm 3.86$ & $6.30 \pm 3.86$ & $0.137^{*}$ & 0.891 & $\mathrm{NS}$ \\
\hline \multicolumn{2}{|c|}{ Platelet $\left(\mathrm{x} 10^{3}\right) / \mathrm{ml}$} & $85.09 \pm 33.87$ & $92.34 \pm 44.34$ & $0.582^{*}$ & 0.560 & $\mathrm{NS}$ \\
\hline \multicolumn{2}{|c|}{ Albumin (g/dl) } & $2.54 \pm 0.67$ & $2.48 \pm 0.66$ & $0.368^{*}$ & 0.713 & $\mathrm{NS}$ \\
\hline \multicolumn{2}{|c|}{ Bilirubin (mg/dl) } & $2.34 \pm 1.45$ & $2.19 \pm 1.51$ & $0.973^{*}$ & 0.330 & $\mathrm{NS}$ \\
\hline \multicolumn{2}{|c|}{ GOT (IU/ml). } & $58.94 \pm 33.13$ & $61.17 \pm 35.29$ & $0.228 *$ & 0.820 & $\mathrm{NS}$ \\
\hline \multicolumn{2}{|c|}{ GPT (IU/ml) } & $50.03 \pm 31.51$ & $52.63 \pm 38.46$ & $0.085^{*}$ & 0.933 & $\mathrm{NS}$ \\
\hline \multicolumn{2}{|l|}{ PT $(\mathrm{sec})$} & $16.83 \pm 3.42$ & $16.72 \pm 3.42$ & $0.137^{*}$ & 0.891 & $\mathrm{NS}$ \\
\hline \multicolumn{2}{|l|}{ INR } & $1.49 \pm 0.35$ & $1.46 \pm 0.32$ & $0.251 *$ & 0.802 & $\mathrm{NS}$ \\
\hline \multicolumn{2}{|l|}{ APRI score } & $1.89 \pm 1.08$ & $1.83 \pm 1.09$ & $0.319^{*}$ & 0.750 & $\mathrm{NS}$ \\
\hline
\end{tabular}

\# Chi-square $\cdot$ independent $t$ test *Mann-Whitney U test, NS non significant 
Table (3): Endoscopic findings in both groups at the beginning of the study and after two weeks

\begin{tabular}{|c|c|c|c|c|c|c|c|c|}
\hline & \multicolumn{2}{|c|}{$\begin{array}{l}\text { Group I } \\
\text { No.=52 }\end{array}$} & \multicolumn{2}{|c|}{$\begin{array}{c}\text { Group II } \\
\text { No.=52 }\end{array}$} & \multirow[t]{2}{*}{$\mathbf{X} 2$} & \multirow[t]{2}{*}{$\mathbf{P}$} & \multirow[t]{2}{*}{ Sig. } \\
\hline & & No. & $\%$ & No. & $\%$ & & & \\
\hline \multirow{2}{*}{ Risky signs } & Absent & 11 & 21.2 & 8 & 15.4 & \multirow[t]{2}{*}{0.58} & \multirow[t]{2}{*}{0.446} & \multirow[t]{2}{*}{$\overline{\mathrm{NS}}$} \\
\hline & Present & 41 & 78.8 & 44 & 84.6 & & & \\
\hline \multirow{3}{*}{$\begin{array}{l}\text { No. of } \quad \text { Oesophageal } \\
\text { varices cords }\end{array}$} & 2 & 18 & 34.6 & 17 & 32.75 & 0.57 & 0.449 & $\mathrm{NS}$ \\
\hline & 3 & 27 & 51.9 & 27 & 51.9 & 0.73 & 0.394 & NS \\
\hline & 4 & 7 & 13.4 & 8 & 15.4 & 0.06 & 0.811 & $\mathrm{NS}$ \\
\hline \multirow{4}{*}{$\begin{array}{l}\text { Oesophageal varices(OV) } \\
\text { grade }\end{array}$} & OV I & 0 & 0 & 0 & 0 & \multirow[t]{4}{*}{0.04} & \multirow[t]{4}{*}{0.847} & \multirow[t]{4}{*}{$\overline{\mathrm{NS}}$} \\
\hline & OV II & 16 & 30.7 & 15 & 28.8 & & & \\
\hline & OV III & 24 & 46.2 & 26 & 50 & & & \\
\hline & OV IV & 8 & 15.4 & 9 & 17.3 & & & \\
\hline \multicolumn{2}{|l|}{$\begin{array}{l}\text { Amount of EO (cc) } \\
\text { Mean } \pm \text { SD }\end{array}$} & \multicolumn{2}{|c|}{$10.2 \pm 4.3$} & \multirow{2}{*}{\multicolumn{2}{|c|}{$5.1 \pm 0.9$}} & \multirow{2}{*}{ 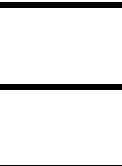 } & \multirow{2}{*}{ 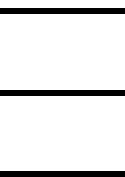 } & \\
\hline \multicolumn{2}{|l|}{$\begin{array}{l}\text { Number of rubber bands } \\
\text { Mean } \pm \text { SD }\end{array}$} & & & & & & & \\
\hline \multirow[t]{3}{*}{ PHG grade } & PHGI & 4 & 7.69 & 3 & 5.76 & 0.29 & 0.593 & $\mathrm{NS}$ \\
\hline & PHGII & 23 & 44.2 & 22 & 42.3 & 0.04 & 0.833 & $\overline{N S}$ \\
\hline & PHGIII & 23 & 44.2 & 25 & 48.1 & 0.16 & 0.689 & NS \\
\hline \multicolumn{2}{|l|}{ Duodenopathy } & 16 & 30.7 & 18 & 34.6 & 0.24 & 0.628 & $\mathrm{NS}$ \\
\hline
\end{tabular}

NS non significant

Table (4): Post endoscopy symptoms

\begin{tabular}{|l|c|c|c|c|c|c|c|}
\hline \multirow{2}{*}{} & \multicolumn{2}{|c|}{$\begin{array}{c}\text { Group I } \\
\text { No.=50 }\end{array}$} & \multicolumn{2}{c|}{$\begin{array}{c}\text { Group II } \\
\text { No.=50 }\end{array}$} & \multirow{2}{*}{ X2 } & \multirow{2}{*}{ P } & \multirow{2}{*}{ Sig. } \\
\cline { 2 - 6 } & No. & $\mathbf{\%}$ & No. & $\mathbf{\%}$ & & & \\
\hline Dysphagia & 35 & 67.3 & 32 & 61.5 & 0.17 & 0.68 & NS \\
\hline Epigastric pain & 47 & 90.3 & 40 & 76.9 & 1.78 & 0.182 & NS \\
\hline Heart burn & 40 & 76.9 & 38 & 73.1 & 0.23 & 0.629 & NS \\
\hline Odynophagia & 29 & 55.7 & 33 & 63.4 & 0.68 & 0.409 & NS \\
\hline Retrosternal pain & 47 & 90.3 & 41 & 78.8 & 1.82 & 0.186 & NS \\
\hline Dyspepsia & 45 & 86.5 & 39 & 75 & 1.96 & 0.161 & NS \\
\hline
\end{tabular}

NS non significant

Table (5): Rates of rebleeding and mortality

\begin{tabular}{|c|c|c|c|c|c|c|c|c|}
\hline & \multicolumn{2}{|c|}{$\begin{array}{c}\text { Group I } \\
\text { No=52 }\end{array}$} & \multicolumn{2}{|c|}{$\begin{array}{c}\text { Group II } \\
\text { No=52 }\end{array}$} & \multirow[t]{2}{*}{$\mathbf{X} 2$} & \multirow[t]{2}{*}{$\mathbf{P}$} & \multirow[t]{2}{*}{ Sig. } \\
\hline & & No & $\%$ & No & $\%$ & & & \\
\hline \multirow[t]{5}{*}{ Rebleeding } & 2 weeks & 5 & 9.5 & 3 & 5.76 & 2.17 & 0.14 & NS \\
\hline & 4 weeks & 2 & 4.25 & 1 & 2.04 & 2.05 & 0.81 & $\mathrm{NS}$ \\
\hline & 6 weeks & 1 & 2.22 & 1 & 2.22 & 0 & 1 & $\mathrm{NS}$ \\
\hline & No & 44 & $84.6 \%$ & 47 & $90.4 \%$ & \multirow{2}{*}{0.791} & \multirow{2}{*}{0.374} & \multirow{2}{*}{ NS } \\
\hline & Yes & 8 & $15.4 \%$ & 5 & $9.6 \%$ & & & \\
\hline \multirow[t]{4}{*}{ Cause } & Ulcers & 5 & $62.5 \%$ & 3 & $60 \%$ & 0.008 & 0.928 & $\mathrm{NS}$ \\
\hline & PHG & 2 & $25 \%$ & 1 & $20 \%$ & 0.043 & 0.835 & $\mathrm{NS}$ \\
\hline & $\mathrm{OV}$ & 1 & $12.5 \%$ & 0 & $0 \%$ & 0.677 & 0.411 & $\mathrm{NS}$ \\
\hline & $\mathrm{GV}$ & 0 & $0 \%$ & 1 & $20 \%$ & 1.733 & 0.188 & $\mathrm{NS}$ \\
\hline \multirow[t]{2}{*}{ Mortality } & Survival & 41 & $79 \%$ & 40 & $79.8 \%$ & \multirow{2}{*}{0.210} & \multirow{2}{*}{0.647} & \multirow{2}{*}{ NS } \\
\hline & Death & 11 & $21 \%$ & 10 & $19.2 \%$ & & & \\
\hline \multirow[t]{2}{*}{ Mortality after rebleeding } & Survival & 5 & $62.5 \%$ & 3 & $60 \%$ & \multirow{2}{*}{0.008} & \multirow{2}{*}{0.928} & \multirow{2}{*}{ NS } \\
\hline & Death & 3 & $37.5 \%$ & 2 & $40 \%$ & & & \\
\hline
\end{tabular}

NS non significant 
Table (6): Correlation between rebleeding and selected study parameters

\begin{tabular}{|c|c|c|c|c|c|c|}
\hline & \multicolumn{2}{|c|}{$\begin{array}{l}\text { Group I } \\
(\mathrm{N}=52)\end{array}$} & \multicolumn{2}{|c|}{$\begin{array}{c}\text { Group II } \\
(\mathrm{N}=52)\end{array}$} & \multicolumn{2}{|c|}{$\begin{array}{l}\text { All studied cases } \\
(\mathrm{N}=104)\end{array}$} \\
\hline & $\mathbf{r}$ & P (Sig.) & $\mathbf{r}$ & p(Sig.) & $\mathbf{r}$ & P (Sig.) \\
\hline Age & +0.016 & $\begin{array}{l}0.910 \\
(\mathrm{NS})\end{array}$ & +0.148 & $\begin{array}{l}0.296 \\
(\mathrm{NS})\end{array}$ & +0.048 & $\begin{array}{c}0.625 \\
(\mathrm{NS})\end{array}$ \\
\hline Sex (Male, Female) & +0.062 & $\begin{array}{l}0.661 \\
(\mathrm{NS})\end{array}$ & +0.023 & $\begin{array}{l}0.869 \\
(\mathrm{NS})\end{array}$ & +0.038 & $\begin{array}{l}0.698 \\
(\mathrm{NS})\end{array}$ \\
\hline Diabetes (No, Yes) & +0.144 & $\begin{array}{l}0.308 \\
(\mathrm{NS})\end{array}$ & +0.042 & $\begin{array}{l}0.769 \\
(\mathrm{NS}) \\
\end{array}$ & +0.093 & $\begin{array}{l}0.347 \\
(\mathrm{NS}) \\
\end{array}$ \\
\hline Hypertension (No, Yes) & +0.277 & $\begin{array}{l}0.347 \\
(\mathrm{NS})\end{array}$ & +0.118 & $\begin{array}{l}0.406 \\
(\mathrm{NS})\end{array}$ & +0.074 & $\begin{array}{l}0.456 \\
(\mathrm{NS})\end{array}$ \\
\hline Bilharzial antigen & +0.434 & $0.326(\mathrm{NS})$ & +0.376 & $\begin{array}{l}0.287 \\
(\mathrm{NS})\end{array}$ & +0.334 & $\begin{array}{l}0.187 \\
(\mathrm{NS})\end{array}$ \\
\hline Primary prophylaxis & -0.393 & $\begin{array}{l}0.295 \\
(\mathrm{NS})\end{array}$ & -0.243 & $\begin{array}{l}0.182 \\
(\mathrm{NS})\end{array}$ & -0.274 & $\begin{array}{l}0.187 \\
(\mathrm{NS})\end{array}$ \\
\hline Ascites(Absent, Mild,...) & +0.192 & $\begin{array}{l}0.173 \\
(\mathrm{NS})\end{array}$ & +0.126 & $\begin{array}{l}0.374 \\
(\mathrm{NS})\end{array}$ & +0.163 & $\begin{array}{l}0.099 \\
(\mathrm{NS})\end{array}$ \\
\hline $\begin{array}{l}\text { Child classification }(\mathrm{A}, \mathrm{B} \text {, } \\
\mathrm{C})\end{array}$ & +0.145 & $\begin{array}{l}0.078 \\
(\mathrm{NS})\end{array}$ & +0.123 & $\begin{array}{l}0.093 \\
(\mathrm{NS})\end{array}$ & +0.136 & $\begin{array}{c}0.014 \\
(\mathrm{~S})\end{array}$ \\
\hline PT & +0.436 & $\begin{array}{l}0.001 \\
(\mathrm{HS})\end{array}$ & +0.250 & $\begin{array}{c}0.044 \\
(\mathrm{~S})\end{array}$ & +0.350 & $\begin{array}{c}<0.001 \\
(\mathrm{HS})\end{array}$ \\
\hline INR & +0.526 & $\begin{array}{c}<0.001 \\
(\mathrm{HS})\end{array}$ & +0.283 & $\begin{array}{c}0.042 \\
(\mathrm{~S})\end{array}$ & +0.419 & $\begin{array}{c}<0.001 \\
(\mathrm{HS})\end{array}$ \\
\hline $\mathrm{OV} \operatorname{grade}(2,3,4)$ & +0.259 & $\begin{array}{c}0.008 \\
(\mathrm{HS})\end{array}$ & +0.207 & $\begin{array}{c}0.024 \\
(\mathrm{~S}) \\
\end{array}$ & +0.233 & $\begin{array}{l}0.001 \\
(\mathrm{HS})\end{array}$ \\
\hline Risk signs & +0.221 & $\begin{array}{l}0.007 \\
(\mathrm{HS}) \\
\end{array}$ & +0.139 & $\begin{array}{c}0.045 \\
(\mathrm{~S})\end{array}$ & +0.179 & $\begin{array}{l}0.001 \\
(\mathrm{HS}) \\
\end{array}$ \\
\hline Units of blood (0-5) & +0.377 & $\begin{array}{c}0.006 \\
(\mathrm{HS})\end{array}$ & +0.393 & $\begin{array}{l}0.004 \\
(\mathrm{HS})\end{array}$ & +0.387 & $\begin{array}{c}<0.001 \\
(\mathrm{HS})\end{array}$ \\
\hline Hepatic a. RI & +0.126 & $\begin{array}{c}0.372 \\
(\mathrm{NS})\end{array}$ & +0.055 & $\begin{array}{c}0.701 \\
(\mathrm{NS}) \\
\end{array}$ & +0.096 & $\begin{array}{l}0.332 \\
(\mathrm{NS}) \\
\end{array}$ \\
\hline PV velocity & -0.293 & $\begin{array}{c}0.095 \\
(\mathrm{NS})\end{array}$ & -0.243 & $\begin{array}{l}0.082 \\
(\mathrm{NS})\end{array}$ & -0.264 & $\begin{array}{l}0.067 \\
(\mathrm{NS})\end{array}$ \\
\hline PV diameter & +0.090 & $\begin{array}{l}0.527 \\
(\mathrm{NS})\end{array}$ & +0.083 & $\begin{array}{l}0.557 \\
(\mathrm{NS}) \\
\end{array}$ & +0.075 & $\begin{array}{c}0.448 \\
(\mathrm{NS}) \\
\end{array}$ \\
\hline Amount of EO injected & +0.329 & $\begin{array}{c}0.017 \\
(\mathrm{~S})\end{array}$ & --- & --- & --- & --- \\
\hline Number of rubber bands & --- & --- & +0.245 & $\begin{array}{c}0.039 \\
(\mathrm{~S}) \\
\end{array}$ & --- & --- \\
\hline APRI score & +0.634 & $0.526(\mathrm{NS})$ & +0.326 & $\begin{array}{c}0.744 \\
(\mathrm{NS})\end{array}$ & +0.389 & $\begin{array}{c}0.697 \\
(\mathrm{NS})\end{array}$ \\
\hline
\end{tabular}

\section{DISCUSSION}

In our study, 104 patients with first attack of variceal bleeding were randomized in two groups; group I (52 patients were treated by endoscopic sclerotherapy, their mean age was 50.1 year, 36 male and 16 female) and group II (52 patients were treated by endoscopic band ligation), their mean age was 49.7 year, 33male and 19 female. There was no significant difference between both groups regarding age and sex. There was no statistically significant difference between the studied groups regarding the cause of chronic liver disease, the majority of patients in both groups have chronic HCV infection, and this is mostly because $\mathrm{HCV}$ is the leading cause of chronic liver diseases in Egypt [15]. 
There was no statistically significant difference between the studied groups regarding post endoscopy symptoms (dysphagia, odynophagia, retrosternal pain, epigastric pain, heart burn and dyspepsia). Oesophageal membrane injuries (erosions or ulcerations) were found in all patients. This agrees with Gimson et al. who found that Complication rates were similar in the two groups [16]. But, this disagrees with Stiegmann et al. who found band ligation to have improved survival and fewer complications [17]. Also, this disagrees with Laine et al. who reported a significant reduction in local complications but no difference in rebleeding or mortality [18]. Moreover, Frequency of treatment induced complications in band ligation were significantly lower as compared with sclerotherapy, mild chest pain and transient fever were significantly more in sclerotheray as reported by Shafqat et al. [19].

In this study, there was no significant difference between the two studied groups as regards rate of early rebleeding. This agrees with Lo et al. who reported that the rate of early rebleeding following EVL was between $9 \%$ and $19 \%$, which is close to results of $\mathrm{Xu}$ et al. who stated that the incidence of early rebleeding following EVL was (7.6\%). Lo et al. reported $17 \%$ rate of rebleeding with band ligation vs. $33 \%$ with sclerptherapy, Villanueva et al., (2006), reported $12 \%$ incidence rate for re-bleeding for band ligaton versus $21 \%$ for sclerotherapy [20-23].

Causes of early rebleeding in the sclerotherapy group were: sclerosant ulcer in 5 cases $(62.5 \%)$, PHG in 2 cases $(25 \%)$ and OV in one case. This agrees with Sauerbruch et al. who found that early rebleeding following sclerotherapy is caused by sclerosant ulcer in most patients [24]. While, causes of early rebleeding in the band ligation group were: post banding ulcer in 3 cases $(60 \%)$, PHG in one case (20\%) and GV in one case $(20 \%)$.This result agrees with Vanbiervliet et al. who reported that cases of severe bleeding after EVL were all caused by early slippage of the rubber bands, leaving the unhealed ulcers. Usually, the bands slip spontaneously within the second week after EVL [25].

Mortality among rebleeding cases in the sclerotherapy group was $37.5 \%$, while mortality in the band ligation group was $40 \%$. The mortality rates in the previous literature ranged between $8 \%$ and $25 \%$. This lower mortality rates are related to the improvement in the endoscopy techniques and in the efficacy of vasoactive drugs and prophylactic antibiotics [26-29].

After two weeks of follow up there was no significant difference between both groups as regards clinical, laboratory data and endoscopic findings. Most cases of early rebleeding occur during the first 2 weeks of follow up. Rebleeding was due to development of sclerosant or post banding ulcers ( 5 cases in the first group and 3 cases in the second group). This agrees with $\mathrm{Xu}$ et al. who found that post-EVL bleeding was most likely to occur between the $7^{\text {th }}$ and $13^{\text {th }}$ day following the procedure [21]. Also, this agrees with Akriviadi et al. who found higher incidence of sclerosant ulcer and rebleeding when endoscopy was repeated earlier, e.g., $70 \%$ at 1 week and 30\% at two week intervals [30]. Also, Tabibian et al. found that most esophageal ulcers bleeding (28 of 32) occurred within 2 weeks after the latest endoscopic treatment [31]. This can be explained by the more complete healing of the ulcer 2 weeks after endoscopic treatment.

After 4 weeks of follow up there was no significant difference between both groups as regards clinical, laboratory data and endoscopic findings. Rebleeding occurs in 2 cases in group I and one case in group II. The cause of rebleeding in both groups was due to severe portal hypertensive gastropathy (PHG). After 6 weeks of follow up there was no significant difference between both groups as regards clinical, laboratory data and endoscopic findings. Rebleeding occurs in one case in group I (due to bleeding OV) and one case in group II (due to bleeding gastric varix).

In our study, it was found that early rebleeding has significant positive correlation with childPugh grade. Also this agrees with Yang et al. (2007) who found that the Child-Pugh score for liver function was an independent risk factor of post-EVL rebleeding [32]. This also agrees with Benedeto-Stojanov et al. who stated that patients with the most severe hepatocellular dysfunction (Child's group C) have the shortest period between the first bleeding and rebleeding (mean 20.8 days) [33]. Our results agree with Berreta et al. who proved that Child-Pugh $\mathrm{C}$ was an independent risk factor of death from rebleeding [34]. Also, this agrees with Amitrano et al. who concluded that child class $\mathrm{C}$ was an independent predictor of recurrent bleeding; mortality was mainly related to the severity of liver failure. This can be explained by the general concept that 
patients with hepatic decompensation bleed more severely than those without hepatic decompensation $[35,36]$.

But, this disagrees with Zhao JR et al. who found that child class was not correlated with the risk of rebleeding and mortality based on univariate analyses. This difference because he used another procedure in treating bleeding Oesophegeal varices: Percutaneous Trans hepatic variceal embolization (PTVE). During PTVE, the portal vein is catheterized by a percutaneous trans-hepatic approach and the gastric vein feeding the varix is embolized with ethanol, steel coils, or cyanoacrylate glue using multi-detector row computed tomography [27].

Size and extent of esophageal varices seen at index endoscopy were also significantly positively correlated to the rebleeding. This result agrees with Benedeto-Stojanov et al. who found that primary variceal bleeding was present in $50 \%$ patients with medium and in $65.38 \%$ patients with large varices [33]. There was no bleeding in patients with small varices. Also, our result agrees with $\mathrm{Xu}$ et al. who found that the extent and size of varices are independent risk factors for early rebleeding. Varices that extend along the entire esophagus are much more dangerous than varices that are limited to the middle and lower part. Moreover, a greater extent of varices often means that more rubber bands are needed, increasing the possibility of rebleeding [21]. It also agrees with Varghese, et al. who stated that higher grades of varices, presence of cherry-red spots and fundal varices predict variceal bleed in patients with liver cirrhosis [37]. The only exception to this is a study done by Koch et al. who found that $35 \%$ of patients with small varices bled, while only $20 \%$ of patients with large varices also bled. This difference because of small sample size, most cases were child class A and longer duration of follow up (36 months) [38].

In our study; there was significant positive correlation between rebleeding and presence of risky signs on varices. All early rebleeding cases in both groups had risky signs on varices at index endoscopy. This agrees with the study of the Northern Italian Endoscopic Club (NIEC) has shown that endoscopic finding of "red signs" is related to the variceal bleeding [39]. Also, Benedeto-Stojanov et al. has shown that endoscopic finding of "red signs" is related to the variceal bleeding. The "red signs" were found in $85 \%$ of large varices with bleeding [33].
There was positive significant correlation between rebleeding and the amount of EO injected in sclerotherapy group and number of rubber bands used in band ligation group. This agrees with $\mathrm{Xu}$ et al. who found that the number of rubber bands was an independent risk factor for re-bleeding after EVL. Therefore, for varices which were in the mild to moderate class, it may not be reasonable to launch many rubber bands. For severe varices, however, it's usually unavoidable to use more bands [21].

\section{CONCLUSION}

Sclerotherapy is associated with higher incidence of rebleeding than band ligation. Most cases of early rebleeding occur during the first 2 weeks of follow up and were due to development of sclerosant or post banding ulcers. Early rebleeding in both groups was correlated to child pugh classification grade (early rebleeding more in child class C>child class B>child class A), elevated coagulation parameters (elevation in PT, INR) among studied groups, grade of oesophegeal varices: Most cases of early rebleeding cases had esophageal varices grade IV and presence of risky signs on varices. No significant correlation between rebleeding and ascites, PV diameter and color Doppler studies could be detected. No statistically significant difference between endoscopic sclerotherapy and band ligation regarding post endoscopy complications could be detected. No significant differences between scleotherapy and band ligation as regards overall mortality or mortality after rebleeding.

Ethical approval: Approved.

Funding: None.

Conflict of interest: Authors declare no conflict of interest related to this article.

\section{REFERENCES}

1- De Franchis R. Natural history of portal hypertension in patients with cirrhosis. J Clin Liver Dis 2001; 5: 645-63.

2- Jensen DM. Endoscopic screening of varices in cirrhosis: findings, implications and outcomes. $J$. Gastroenterol 2002; 122:1620-30.

3- Cales P, Desmorat H, Vinel JP, Caucanas JP, Ravaud A, Gerin P et al. Incidence of large oesophegeal varices in patients with cirrhosis: application to prophylaxis of first bleeding. Gut 1990; 31: 1298-302. 
4- Krige JE, Shaw JM, Bornman PC. The evolving role of endoscopic treatment for bleeding esophageal varices. World J Surg 2005; 29: 96673.

5- Sharara AI , Rockey DC. Gastro-esophageal variceal hemorrhage. $N$ Engl $J$ Med 2001; 345(9):669-681.

6- Krige J, Kotze U, Distiller G, Shaw JM, Bornman PC. predictive factors for rebleeding and death in alcoholic cirrhotic patients with acute variceal bleeding: multivariate analysis. World J Surg 2009; 33: 2127-2135.

7- Triantos C, Vlachogiannakos J, Armonis A, Saveriadis A, Kougioumtzian A, Leandro G et al. Primary prophylaxis of variceal bleeding in cirrhotics unable to take beta-blockers: a randomized trial of ligation. Aliment Pharmacol Ther 2005; 21:1435-1443.

8- Henderson JM, Barnes DS, Geisinger MA Portal hypertension. Current Problems in Surgery 1998; $35,381-452$.

9- Wai CT, Greenson JK, Fontana RJ, Kalbfleisch JD, Marrero JA, Conjeevaram HS et al. A simple noninvasive index can predict both significant fibrosis and cirrhosis in patients with chronic hepatitis C. Hepatology 2003; 38: 518-526.

10- Schneider AW, Kalk JF, Klein CP. Hepatic arterial pulsatility index in cirrhosis: correlation with portal pressure. J Hepatol 1999; 30: 876881.

11- Child CG and Turcotte JG. Surgery and portal hypertension. In Child CG. The liver and portal hypertension. Philadelphia: Saunders 1964; 5064.

12- Thakeb F. A study of the oesophegus by endoscopy and radiology after sclerotherapy. Gastrointestinal Endoscopy: an Egyptian view. By Thakeb F and Zakaria S (eds.). El Sona ElMohamadia, Egypt 1988, P. 51.

13- Pungpapong S, Keaveny A, Raimondo M, Dickson R, Woodward T, Harnois D et al. Accuracy and interobserver agreement of small-caliber vs. conventional esophagogastro-duodenoscopy for evaluating esophageal varices. Endoscopy 2007; 39(8):673-80.

14- Banares R, Albillos A, Rincón D, Alonso S, González M, Ruiz-del-Arbol L et al. Endoscopic treatment versus endoscopic plus pharmacologic treatment for acute variceal bleeding: a metaanalysis. Hepatology 2002; 35:609-615.

15- Mohamoud YA, Mumtaz GR, Riome S, Miller DW, AbuRadda LJ. The epidemiology of hepatitis $\mathrm{C}$ virus in Egypt: a systematic review and data synthesis. BMC Infectious Diseases 2013; $13: 288$
16- Gimson AE, Ramage JK, Panos MZ, Hayllar K, Harrison PM, Williams R, et al. Randomised trial of variceal banding ligation versus injection sclerotherapy for bleeding oesophageal varices. Lancet 1993; 342:391-4.

17- Stiegmann GV, Goff JS, Michaletz-Onody PA, Korula J, Lieberman D, Saeed ZA et al. Endoscopic sclerotherapy as compared with endoscopic ligation for bleeding esophageal varices. N Engl J Med 1992; 326: 1527-1632.

18- Laine L, el-Newihi HM, Migikovsky B, Sloane R, Garcia F. Endoscopic ligation compared with sclerotherapy for the treatment of bleeding esophageal varices. Ann Intern Med 1993; 119:17.

19- Shafqat F, Khan AA, Alam A, Arshad, Butt K, Shah SW et al. Band Ligation Vs Endoscopi Sclerotherapy in Esophageal Varices: A Prospective Randomized Comparison. The Journal of the Pakistan Medical Association 1998; 48(7):192 -6.

20- Lo GH, Chen WC, Chen MH, Lin CP, Lo CC, Hsu PI, et al. Endoscopic ligation vs. nadolol in the prevention of first variceal bleeding in patients with cirrhosis. Gastrointest Endosc 2004; 59:333- 338 .

21- Xu L, Ji F, Xu QW, Zhang MQ. Risk factors for predicting early variceal rebleeding after endoscopic variceal ligation. World. $J$ Gastroenterol 2011; 17(28): 3347-3352.

22- Lo GH, Lai KH, Cheng JS, Lin CK, Huang JS, Hsu PI, et al. Emergency banding ligation versus sclerotherapy for the control of active bleeding from esophageal varices. Hepatology 1997; 25 :1101-1104.

23- Villanueva C, Piqueras M, Aracil C, Gómez C, López-Balaguer JM, Gonzalez B, et al. A randomized controlled trial comparing ligation and sclerotherapy as emergency endoscopic treatment added to somatostatin in acute variceal bleeding. Journal of Hepatology 2006; 45, 560567.

24- Sauerbruch T, Weinzierl M, Kopcke W, Paumgartner G. Long-term sclerotherapy of bleeding esophageal varices in patients with liver cirrhosis. An evaluation of mortality and rebleeding risk factors. Scand J Gastroenterol 1985; 20: 51-58.

25- Vanbiervliet G, Giudicelli-Bornard S, Piche T, Berthier F, Gelsi E, Filippi J et al. Predictive factors of bleeding related to post-banding ulcer following endoscopic variceal ligation in cirrhotic patients: a case-control study. Aliment Pharmacol Ther 2010; 32: 225-232. 
26- Luz GO, Filho FM, Matuguma SE, Hondo FY, Ide E, Melo JM et al. Comparison between endoscopic sclerotherapy and band ligation for hemostasis of acute variceal bleeding. World $J$ Gastrointest Endosc 2011; 16; 3(5): 95-100.

27- Zhao JR,Wang GC ,Hu JH, Zhang CQ. Risk factors for early rebleeding and mortality in acute variceal hemorrhage. World J Gastroenterol 2014; 20(47): 17941-17948.

28- Villanueva C, Aracil C, Colomo A, LopezBalaguer JM, Piqueras M, Gonzalez B et al. Clinical trial: a randomized controlled study on prevention of variceal rebleeding comparing nadolol + ligation vs. hepatic venous pressure gradient-guided pharmacological therapy. Aliment Pharmacol Ther 2009; 29: 397-408.

29- Bosch J, Abraldes JG, Groszmann RJ. Current management of portal hypertension. J Hepatol 2003; 38:S54-S68.

30- Akriviadi SE, Korula A, Gupta S, Ko Y, Yamada S. Frequent endoscopic variceal sclerotherapy increases risk of complications Prospective randomized controlled study of two treatment scheduks. Dig Dus 1989; Scu 34 1068-74.

31- Tabibian N, Smith LJ, Graham DY. Sclerotherapy-associated esophageal ulcers: lessons from a double-blind, randomized comparison of sucralfate suspension versus placebo. Gastrointest Endosc 1989; 35:312-5.

32- Yang MT, Chen HS, Lee HC, Lin CL. Risk factors and survival of early bleeding after esophageal variceal ligation. Hepatogastroenterology 2007; 54: 1705-170.

33- Benedeto-Stojanov D, Nagorni A, Mladenović B, Stojanov D, Denić N. Risk and causes of gastroesophageal bleeding in patients with liver cirrhosis. Vojnosanit Pregl 2007; 64(9):585-9.
34- Berreta J, Kociak D, Corti R, Morales G, Ortiz M, Laplacette $\mathrm{M}$ et al. Predictors of intrahospitalary mortality in the upper gastrointestinal variceal bleeding due to chronic liver disease treated endoscopically. Acta Gastroenterol Latinoam 2008; 38: 43-500

35- Amitrano L, Guardascione MA, Manguso F, Bennato R, Bove A, DeNucci $\mathrm{C}$ et al. The effectiveness of current acute variceal bleed treatments in unselected cirrhotic patients: refining short-term prognosis and risk factors. Am J Gastroenterol 2012; 107: 1872-1878.

36- Baveno T. Updating Consensus in portal hypertension: report of the Baveno III consensus Workshop on definitions, methodology and therapeutic strategies in portal hypertension. Journal of Hepatology 2010; 33:846-825.

37- Varghese J, Cherian J V, Solomon R, Jayanthi V. Predictors of variceal bleed among patients with liver cirrhosis in the era of sclerotherapy .Singapore Med J 2008; 49(3): 239-242.

38- Koch H, Henning H, Grimm H, Soehendra N. Prophylactic sclerosing of oesophageal varices results of a prospective controlled study. Endoscopy 1986;18: 40-3.

39- North Italian Endoscopic Club. Prediction of the first variceal hemorrhage in patients with cirrhosis of the liver and esophageal varices: a prospective multicenter study. The North Italian Endoscopic Club for the Study and Treatment of Esophageal Varices. $N$ Engl J Med 1988; 319:983-989.

Peer reviewer: Amira Suliman, Professor of Tropical Medicine and Hepatogastroenterology, Faculty of Medicine, Zagazig University, Egypt. Editor: Mohamad Emara, Assistant Professor of Tropical Medicine and Hepatogastroenterology, Faculty of Medicine, Zagazig University, Egypt 